\title{
CCR9+ and CD103+ tolerogenic dendritic cell populations in food allergy patients undergoing oral immunotherapy
}

\author{
Marco Garcia ${ }^{1}$, Amit Singh², Grace Yu ${ }^{3}$, Robi Bucayu ${ }^{3}$, Trevor Longbottom ${ }^{3}$, Kari Nadeau ${ }^{*}$ \\ From Food Allergy and Anaphylaxis Meeting 2011 \\ Venice, Italy. 17-19 February 2011
}

\section{Rationale}

CD103+ DCs (dendritic cells) and CCR9+ pDCs (plasmacytoid DCs) have been implicated in promoting tolerance to antigens through regulatory-T cell induction. We have conducted food oral immunotherapy (OIT) clinical studies for the last 3 years at Stanford University. We hypothesized that subjects with food allergies have low CD103+ and CCR9+ expression on their DCs but that these DC populations change over time while on therapy.

\section{Methods}

OIT was conducted and blood samples were drawn at baseline and approximately every 5 months during the study. The study is currently ongoing. PBMCs (peripheral blood mononuclear cells) were purified and flow cytometry was performed on gated DCs (LSRII, BD Biosciences).

\section{Results}

DCs expressing CD103 (integrin-alpha E) and CCR9 (CCL25 chemokine receptor) were examined in three cohorts - (1) patients undergoing milk or peanut OIT $(\mathrm{n}=8)$, (2) healthy controls $(\mathrm{HC})(\mathrm{n}=8)$, and (3) non-OIT food allergy patients (FA) $(n=8)$. PBMCs were incubated for 6 or 18 hours either with or without offending allergen. CCR9+ expression on pDCs was significantly greater in $\mathrm{HC}$ versus FA patients $(42 \% \pm 25 \%$ vs $11 \%$ $\pm 10 \%$; $\mathrm{p}<0.01$ ) while CD103 expression on DCs was comparatively greater in $\mathrm{HC}$ versus FA patients $(0.19 \%$ $\pm 0.17 \%$ vs $0.07 \% \pm 0.07 \% ; p=0.16$ ). After offending allergen stimulation for both 6 and 18 hours, CCR9 presence on pDCs significantly increased more in FA patients than in HC patients (213MFI*-6hr, 188MFI-18hr versus 12MFI for HC; $\mathrm{p}<0.03)$. In OIT patients, CCR9 change on pDCS after stimulation was significantly different than their baseline CCR9 MFI shift values (16MFI versus $188 \mathrm{MFI} ; \mathrm{p}<0.03)$ and more in line with the HC profile. "-Median Fluorescent Intensity.

\section{Conclusion}

The CCR9 and CD103 DC populations may play an important role for food allergy patients undergoing OIT. These tolerogenic DC changes in OIT may reveal one way that regulatory $\mathrm{T}$-cell mediated tolerance, $\mathrm{T}$-cell anergy, and/ or clonal deletion is induced.

\section{Author details}

${ }^{1}$ Stanford University, Biology, Stanford, USA. ${ }^{2}$ Stanford University, Allergy and Immunology, Stanford, USA. ${ }^{3}$ Stanford University, Pediatrics, Stanford, USA.

Published: 12 August 2011

doi:10.1186/2045-7022-1-S1-051

Cite this article as: Garcia et al: CCR9+ and CD103+ tolerogenic dendritic cell populations in food allergy patients undergoing oral immunotherapy. Clinical and Translational Allergy 2011 1(Suppl 1):051.

2Stanford University, Allergy and Immunology, Stanford, USA

Full list of author information is available at the end of the article

C 2011 Garcia et al; licensee BioMed Central Ltd. This is an open access article distributed under the terms of the Creative Commons 